\title{
Herd Immunity as a hope for protection against COVID-19 virus and other life-threatening infections
}

\author{
Moustafa Abdelnasser, PhD (Microbiol), MHPE, Diploma (TQM) \\ mostafa_online1@yahoo.com, mostafa.nasser@azhar.edu.eg
}

\begin{abstract}
Since the emergence of SARS-Cov2 (COVID-19) virus in December 2019, there is a dilemma in defining the ways of protection and therapy of this new strain of Coronavirus family. Many trials have been taken to protect healthy individuals from contracting infection. Basic infection control measures were applied. These include frequent hand hygiene, wearing respirator (e.g. N95) surgical or cloth mask, cough etiquette and adherence to physical distance (1-2 meters between two individuals). Plasma from recovered patients was tried with COVID-19 patients with severe conditions. In March 2020, World Health Organization (WHO) announced that COVID-19 became pandemic. Meanwhile, there has been a call for lockdown in different countries all over the world with limitation of working hours or working from home with virtual meeting. We are now in the $3^{\text {rd }}$ wave of this aggressive virus. The rational of this editorial is that until now infection control measures are a comprehensive trial to mitigate or stop the spread of infections on the community level. We need a more stringent and effective way to stop the spread of the virus at the portal of entry. Vaccine development was in the agenda of WHO, Center of Disease Control and Prevention (CDC) and pharmaceutical companies from the early beginning of COVID-19 emergence. Our aim was to clarify the role of community protection (herd immunity) in prevention of COVID-19 infection by universal introduction of vaccination policy.
\end{abstract}

\section{INTRODUCTION}

In December 2019, the new corona virus strain or SARS-Cov2 has emerged in Wuhan City of Hupei, Province, China, and in March the WHO declared that there is a pandemic. Travel limitations and lookdown were called for. Patients with severe or moderate symptoms were treated at hospital. Guidelines were issued by WHO, CDC and some national authorities. When more cases started to increase, a census has been taken to treat only severe cases in hospitals and mild or moderated cases at home. Patients recovered from Covid-19were tested for estimated levels of antibodies in their blood. Sera of these patients have been found to contain variable levels of SARS-Cov2. Unfortunately, some patients had low levels of these antibodies with short or low immunity. Moreover, some patients developed a second or even third attack of virus infection. This means 
that there is a controversy in the role of these antibodies in neutralizing virus infection (CDC. 2020a and WHO, 2020).

\section{Herd Immunity}

Herd immunity, also known as population or community immunity, is the indirect protection from an infectious disease that happens when a population is immune either through vaccination or immunity developed through previous infection. WHO supports achieving herd immunity through vaccination, not by allowing a disease to spread through any segment of the population, as this would result in unnecessary cases and deaths. So, those who have a good immunity will resist infection while those with weak immunity as old individuals or with chronic diseases or life-threatening conditions will not resist. This idea was actually based on experience with similar situations of other infectious agents and because it was difficult to order for lockdown for a long time as people have to practice their daily life to some extent (CDC. $2020 \mathrm{a}$ and WHO, 2020).

Herd immunity occurs when a large portion of a community (the herd) becomes immune to a disease, making the spread of disease from person to person unlikely. As a result, the whole community becomes protected - not just those who are immune. Often, a percentage of the population must be capable of getting a disease for it to spread. This is called a threshold proportion. If the proportion of the population that is immune to the disease is greater than this threshold, the spread of the disease will decline. This is known as the herd immunity threshold. Percentage of a community needs to be immune to achieve herd immunity varies from disease to disease. The more contagious a disease is, the greater the proportion of the population that needs to be immune to the disease to stop its spread, e.g. measles is a highly contagious illness. It's estimated that $94 \%$ of the population must be immune to interrupt the chain of transmission (Association of Professional of Infection Control, APIC 2021 and Dong et al.,2021).

\section{There are two ways herd immunity can happen, i.e. infection and vaccines.}

Resistance can develop naturally when the body is exposed to a virus or bacteria. Herd immunity can be reached when enough people in the population have recovered from a disease and have developed protective antibodies against future infection. However, there are some major problems with relying on community infection to create herd immunity to the virus that causes COVID19.

It is not clear how long protection from COVID-19 occurs. Even if there are antibodies, it is possible that COVID-19 infection occurs again. 
Herd immunity also can be reached when enough people have been vaccinated against a disease and have developed protective antibodies against future infection. Vaccines can also build resistance. Unlike the natural infection method, vaccines create immunity without causing illness or resulting complications. Using the concept of herd immunity, vaccines have successfully controlled contagious $(W H O, 2021)$. Vaccines train the immune systems to create proteins that fight disease, known as 'antibodies', just as would happen when we are exposed to a disease but - crucially - vaccines work without making us sick. Like natural infection, herd immunity makes it possible to protect the population from a disease, including those who cannot be vaccinated, such as new-borns or those who have compromised immune systems. Reaching herd immunity through vaccination against COVID-19 might be difficult for many reasons (Aschwanden, 2021and APIC,2021).

- Vaccine hesitancy: Some people may object to getting a COVID19 vaccine because of religious objections, fears about the possible risks or scepticism about the benefits. If the proportion of vaccinated people in a community is below the herd immunity threshold, a contagious disease could continue to spread.

- Protection questions: It is not clear how long the COVID-19 vaccines will protect from COVID-19. Further research is needed to see how much the COVID-19 vaccines reduce transmission of the COVID-19 virus. Also, research suggests that COVID-19 vaccines may have lower efficacy against some of the variants of the COVID-19 virus. New variants, which could be more resistant to vaccines, are regularly emerging.

- Uneven vaccine roll-out: The distribution of COVID-19 vaccines has greatly varied among and within countries. If one community achieves a high COVID-19 vaccination rate and surrounding areas do not, outbreaks can occur if the populations mix.

- Vaccines protect against diseases, but they can also cause a diverse range of mild to severe complications. The most common complications, e.g., soreness, swelling, fever, and rash are mild. Severe complications such as critical allergic reactions or seizures may also be scarcely induced. If these problems occur, they usually begin soon after the shot time and last 1 or 2 days. More serious problems following a flu shot can include small increased risk of Guillain-Barré Syndrome (GBS) - the acute immunemediated polyneuropathies after inactivated flu vaccine $(C D C, 2020 b)$.

The vaccine life cycle passed into four phases of development and approval, preceded by research. Safety is the priority at each phase. There are three stages, i.e. vaccine development, approval and monitoring. The stage of vaccine development includes basic research, discovery, and pre-clinical stages. The second stage includes clinical studies/trials which are; phase I (for safety), phase 
II (for effectiveness), phase 1II (for safety and effectiveness). Monitoring is followed by CDC and Food and Drug Administration (FDA) in phase IV for safety, effectiveness and unexpected adverse effects (CDC. 2020a; FDA, 2020) and WHO, 2021).

\section{COVID Vaccine Trials}

Many trails for COVID-19 vaccine development were carried out in many research centres in USA, UK, China and Russia. On January 29, 2021, after a recommendation from the European Medicines Agency (EMA), the European Commission granted the vaccine "conditional marketing authorization" in the European Union for adults aged 18 years and older. The WHO trusted source issued the vaccine "emergency use listing" on February 15 of year2021(Lurie, et al. 2020and WHO, 2021).

Different types of Covid-19 are now in the market. Some of them are killed virus, others are in the form of viral messenger RNA (Pfizer-Biontic. Moderna) or recombinant Adenovirus with viral genome, i.e. viral vector (Oxford/AstraZeneca, Janssen ( $\mathrm{J} \& \mathrm{~J}$ ), and Gamaleya-Sputnik). This viral vector vaccine contains the gene that encodes for the spike protein on the surface of the SARS-CoV-2 virus. Once delivered to our cells, the gene is transcribed, prompting our cells to make the spike protein. The presence of this protein triggers the body's immune system to produce antibodies to fight against the spike protein, which then prepares the body to fight against SARS-CoV2.Another type of Covid-19 vaccination from China includes the inactivated (killed) virus (Sinovac, Sinopharm). In May 13, 2021, CDC stated that fully vaccinated people no longer need to wear a mask or physically distance in any setting, except where required by federal, state, local, tribal, or territorial laws, rules, and regulations, including local business and workplace guidance. Also, fully vaccinated people can refrain from testing following a known exposure unless they are residents or employees of a correctional or detention facility or a homeless shelter (CDC. 2021 and $W H O, 2021)$.

\section{Future Prospective}

It is now clear that there is a vaccine hesitancy. Like what happens and still from the COVID virus strain and some other have a phobia and do not practice any activity outside home. Similarly, some people do not believe in any new COVID vaccine and others are afraid of their adverse effects. Under these 
situations, we cannot precast what will happen in the near future. Hopefully, we can say that "the more vaccinated people, the less chance to catch or spread Covid-19 infection'. Of course, this sure for many other viral or bacterial vaccines.

\section{REFERENCES}

Aschwanden C. (2021):Five reasons why COVID herd immunity is probably impossible. Nature.591, 520-522.

Association of Professional of Infection Control, APIC(2021): Herd immunity

Center of Disease Control and Prevention CDC (2020a): Corona virus Disease 2019 (COVID-19).

Center of Disease Control and Prevention CDC (2020b): Possible Side effects from Vaccines.

Center of Disease Control and Prevention CDC (2021): Benefits of Getting a COVID-19 Vaccine.

Dong M. He F. and Deng Y. (2021):.How to Understand Herd Immunity in the Context of COVID-19. Viral Immunology34 (3). 174-181. https://doi.org/10.1089/vim.2020.0195.

Food and Drug Administration (FDA) (2020): Guidance for industry: development and licensure of vaccines to prevent COVID-19.

Lurie N., Saville M, Hatchett R and Halton J. (2020): Developing Covid-19 Vaccines at Pandemic Speed. New England Journal of Medicine. 382:1969-1973

World Health Organization (WHO) (2020): Corona virus disease (COVID-19): Herd immunity, lockdowns and COVID-19.

World Health Organization (WHO) (2020): How are vaccines developed?. 